\title{
Study of Quark Confinement in Baryons with Lattice QCD
}

\author{
Hideo Suganuma ${ }^{\mathrm{a}}$, Toru T. Takahashi ${ }^{\mathrm{b}}$, Fumiko Okiharuc ${ }^{\mathrm{c}}$ and Hiroko Ichie ${ }^{\mathrm{a}}$ \\ a Faculty of Science, Tokyo Institute of Technology, Ohokayama 2-12-1, Meguro, Tokyo 152-8551, Japan \\ b Yukawa Institute for Theoretical Physics, Kyoto University, Kitashirakawa, Sakyo 606-8502, Japan \\ ${ }^{c}$ Department of Physics, Nihon University, 1-8 Kanda-Surugadai, Chiyoda, Tokyo 101, Japan
}

In $\mathrm{SU}(3)$ lattice QCD, we perform the detailed study for the ground-state three-quark (3Q) potential $V_{3 \mathrm{Q}}^{\text {g.s. }}$ and the 1 st excited-state $3 \mathrm{Q}$ potential $V_{3 \mathrm{Q}}^{\text {e.s. }}$, i.e., the energies of the ground state and the 1st excited state of the gluon field in the presence of the static three quarks. From the accurate calculation for more than 300 different patterns of $3 \mathrm{Q}$ systems, the static ground-state $3 \mathrm{Q}$ potential $V_{3 \mathrm{Q}}^{\text {g.s. }}$ is found to be well described by the Coulomb plus Y-type linear potential (Y-Ansatz) within 1\%-level deviation. As a clear evidence for Y-Ansatz, Y-type fluxtube formation is actually observed on the lattice in maximally-Abelian projected QCD. For about 100 patterns of $3 \mathrm{Q}$ systems, we calculate the 1 st excited-state $3 \mathrm{Q}$ potential $V_{3 \mathrm{Q}}^{\mathrm{e} . \mathrm{s}}$, and find a large gluonic-excitation energy $\Delta E_{3 \mathrm{Q}} \equiv V_{3 \mathrm{Q}}^{\text {e.s. }}-V_{3 \mathrm{Q}}^{\text {g.s. }}$ of about $1 \mathrm{GeV}$, which gives a physical reason of the success of the quark model even without gluonic excitations. We present also the first study for the penta-quark potential $V_{5 \mathrm{Q}}$ in lattice QCD, and find that $V_{5 \mathrm{Q}}$ is well described by the sum of the OGE Coulomb plus multi-Y type linear potential.

\section{Introduction}

Quantum chromodynamics (QCD), the SU(3) gauge theory, was first proposed by Yoichiro Nambu 11 in 1966 as a candidate for the fundamental theory of the strong interaction, just after the introduction of the "new" quantum number, "color" 2. In spite of its simple form, QCD creates thousands of hadrons and leads to various interesting nonperturbative phenomena such as color confinement [3] and dynamical chiralsymmetry breaking [4]. Even now, it is very difficult to deal with QCD due to its strong-coupling nature in the infrared region.

In recent years, the lattice QCD Monte Carlo calculation becomes a reliable and useful method for the analysis of nonperturbative QCD [5], which indicates an important direction in the hadron physics. In this paper, using lattice QCD, we study the inter-quark potential in detail 6789.

In general, the three-body force is regarded as a residual interaction in most fields in physics. In QCD, however, the three-body force among three quarks is a "primary" force reflecting the $\mathrm{SU}(3)$ gauge symmetry. In fact, the three-quark (3Q) potential is directly responsible for the structure and properties of baryons, similar to the relevant role of the $\mathrm{Q}-\overline{\mathrm{Q}}$ potential for meson properties, and both the $\mathrm{Q}-\overline{\mathrm{Q}}$ potential and the $3 \mathrm{Q}$ potential are equally important fundamental quantities in QCD. Furthermore, the 3Q potential is the key quantity to clarify the quark confinement in baryons. However, in contrast to the Q- $\bar{Q}$ potential [5], there was almost no lattice QCD study for the 3Q potential before our study in 1999 [9], in spite of its importance in the hadron physics.

\section{The Ground-State $3 \mathrm{Q}$ Potential in QCD}

The $\mathrm{Q}-\overline{\mathrm{Q}}$ potential is known to be well described with the inter-quark distance $r$ as 567

$V_{\mathrm{Q} \overline{\mathrm{Q}}}(r)=-\frac{A_{\mathrm{Q} \overline{\mathrm{Q}}}}{r}+\sigma_{\mathrm{Q} \overline{\mathrm{Q}}} r+C_{\mathrm{Q} \overline{\mathrm{Q}}}$.

As for the $3 \mathrm{Q}$ potential form, we note two theoretical arguments at short and long distance limits.

1. At the short distance, perturbative QCD is applicable, and therefore $3 \mathrm{Q}$ potential is expressed as the sum of the two-body Coulomb potential originating from the one-gluon-exchange process.

2. At the long distance, the strong-coupling expansion of QCD is plausible, and it leads to the flux-tube picture [10]. For the 3Q system, there appears a junction which connects the three fluxtubes from the three quarks, and Y-type flux-tube 
formation is expected 6710.

Then, we theoretically conjecture the functional form of the $3 \mathrm{Q}$ potential as the Coulomb plus Y-type linear potential, i.e., Y-Ansatz,

$V_{3 \mathrm{Q}}^{\text {g.s. }}=-A_{3 \mathrm{Q}} \sum_{i<j} \frac{1}{\left|r_{i}-r_{j}\right|}+\sigma_{3 \mathrm{Q}} L_{\min }+C_{3 \mathrm{Q}}$,

where $L_{\min }$ is the minimal value of the total fluxtube length. Of course, it is nontrivial that these simple arguments on UV and IR limits of QCD hold for the intermediate region. Then, we study the $3 \mathrm{Q}$ potential in lattice QCD. Note that the lattice QCD calculation is completely independent of any Ansatz for the potential form.

\subsection{The Three-Quark Wilson Loop}

Similar to the Q-Q potential calculated with the Wilson loop, the $3 \mathrm{Q}$ potential can be calculated with the 3Q Wilson loop 678 defined as

$W_{3 \mathrm{Q}} \equiv \frac{1}{3 !} \epsilon_{a b c} \epsilon_{a^{\prime} b^{\prime} c^{\prime}} U_{1}^{a a^{\prime}} U_{2}^{b b^{\prime}} U_{3}^{c c^{\prime}}$

with $U_{k} \equiv P \exp \left\{i g \int_{\Gamma_{k}} d x_{\mu} A^{\mu}(x)\right\}$ in Fig.1. The $3 \mathrm{Q}$ Wilson loop physically means that a colorsinglet gauge-invariant $3 \mathrm{Q}$ state is created at $t=0$ and is annihilated at $t=T$ with the three quarks spatially fixed for $0<t<T$.

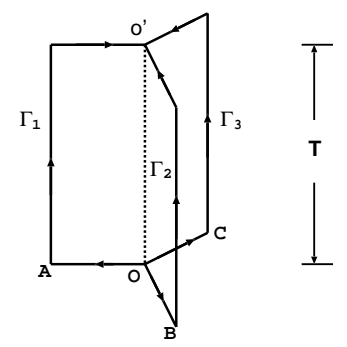

Figure 1. The 3Q Wilson loop $W_{3 \mathrm{Q}}$.

The vacuum expectation value of the $3 \mathrm{Q}$ Wilson loop is expressed as

$\left\langle W_{3 \mathrm{Q}}\right\rangle=\sum_{n=0}^{\infty} C_{n} \exp \left(-V_{n} T\right)$,

where $V_{n}$ denotes the $n$th energy of the gaugefield configuration in the presence of the spatiallyfixed three quarks 678.

While $V_{n}$ depends only on the $3 \mathrm{Q}$ location, $C_{n}$ depends on the operator choice for the $3 \mathrm{Q}$ state.
For the accurate calculation, we need to reduce the excited-state component, i.e., $C_{n}(n \geq 1)$, in the $3 \mathrm{Q}$ state prepared at $t=0$ and $T$.

\subsection{The Smearing Method}

The smearing is a useful method to construct the quasi-ground-state operator in lattice QCD in a gauge-invariant manner, and is actually successful for the ground-state Q- $\overline{\mathrm{Q}}$ potential [7]. (Note that the smearing is just a method to construct the operator, and hence it never changes the gauge configuration unlike the cooling.)

The smeared link-variable $\bar{U}_{\mu}(s)$ includes a spatial extension, and the smeared "line" expressed with $\bar{U}_{\mu}(s)$ corresponds to a Gaussian-distributed "flux-tube" in terms of the original link-variable 77. Therefore, the properly smeared line is expected to resemble the ground-state flux-tube.

Then, through the selection of the properly smeared 3Q Wilson loop $\left\langle W_{3 \mathrm{Q}}\left[\bar{U}_{\mu}(s)\right]\right\rangle$, we can construct the ground-state-dominant $3 \mathrm{Q}$ operator for the accurate measurement of the ground-state $3 \mathrm{Q}$ potential 678.

\subsection{Lattice QCD results for $3 \mathrm{Q}$ Potential}

For more than 300 different patterns of spatially-fixed 3Q systems, we perform the thorough calculation of the ground-state potential $V_{3 \mathrm{Q}}^{\text {g.s. }}$ in $\mathrm{SU}(3)$ lattice $\mathrm{QCD}$ with the standard plaquette action with $12^{3} \times 24$ at $\beta=5.7$ and with $16^{3} \times 32$ at $\beta=5.8$ and 6.0 at the quenched level. For the accurate measurement, we use the smearing method and construct the ground-statedominant 3Q operator 678.

To conclude, we find that the static groundstate $3 \mathrm{Q}$ potential $V_{3 \mathrm{Q}}^{\text {g.s. }}$ is well described by the Coulomb plus Y-type linear potential (Y-Ansatz) within 1\%-level deviation 67.

\subsection{Other Studies on the $3 \mathrm{Q}$ Potential}

To clarify the current status of the $3 \mathrm{Q}$ potential, we introduce recent works of other groups.

de Forcrand's group, who once supported $\Delta$ Ansatz in lattice QCD [11, seems to change their opinion from $\Delta$-Ansatz to Y-Ansatz [12].

Kuzmenko and Simonov also showed that the Delta-shape is impossible from gauge-invariance point of view, and the Y-shaped configuration is the only possible for the three-quark system [13. 
One of the theoretical basis of $\Delta$-Ansatz was Cornwall's conjecture based on the vortex vacuum model [14. Recently, Cornwall re-examined his previous work and found that the correct answer is Y-Ansatz instead of $\Delta$-Ansatz [15].

In this way, Y-Ansatz for the static $3 \mathrm{Q}$ potential seems almost settled both in lattice QCD and in analytic framework.

\subsection{Y-type Flux-Tube Formation}

Recently, as a clear evidence for Y-Ansatz, Ytype flux-tube formation is actually observed in maximally-Abelian (MA) projected lattice QCD from the measurement of the action density in the spatially-fixed 3Q system 1617. (See Fig.2.)
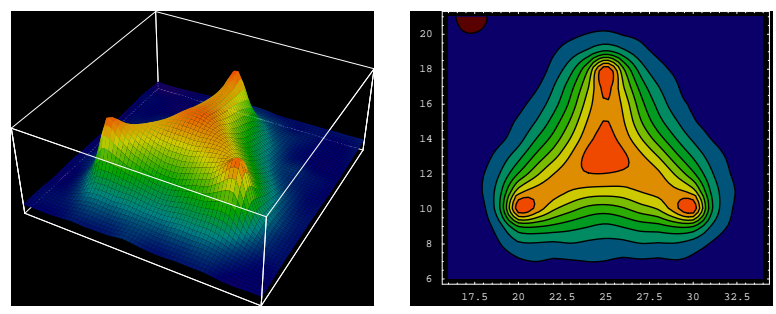

Figure 2. The lattice QCD result for Y-type fluxtube formation in the spatially-fixed 3Q system in MA projected QCD. The distance between the junction and each quark is about $0.5 \mathrm{fm}$.

\section{Penta-Quark Potential in Lattice QCD}

Motivated by the recent discovery of the pentaquark baryon $\Theta^{+}(1540)$, we perform the first study of the static penta-quark (5Q) potential $V_{5 \mathrm{Q}}$ in $\mathrm{SU}(3)$ lattice QCD with $\beta=6.0$ and $16^{3} \times$ 32 at the quenched level. We investigate the QQQ-QQ configuration as shown in Fig.3. With the smearing method 678 to enhance the groundstate component, we accurately calculate the $5 \mathrm{Q}$ potential $V_{5 \mathrm{Q}}$ from the $5 \mathrm{Q}$ Wilson loop $\left\langle W_{5 \mathrm{Q}}\right\rangle$ as shown in Fig.4 in a gauge-invariant manner.

We find that the $5 \mathrm{Q}$ potential $V_{5 \mathrm{Q}}$ is well described by the sum of one-gluon-exchange (OGE) Coulomb term and multi-Y type linear term, which we call the "OGE plus multi-Y Ansatz",

$$
\begin{aligned}
& V_{5 \mathrm{Q}}=\frac{g^{2}}{4 \pi} \sum_{i<j} \frac{T_{i}^{a} T_{j}^{a}}{\left|\mathbf{r}_{i}-\mathbf{r}_{j}\right|}+\sigma_{5 \mathrm{Q}} L_{\min }+C_{5 \mathrm{Q}} \\
& =-A_{5 \mathrm{Q}}\left\{\left(\frac{1}{r_{12}}+\frac{1}{r_{34}}\right)+\frac{1}{2}\left(\frac{1}{r_{15}}+\frac{1}{r_{25}}+\frac{1}{r_{35}}+\frac{1}{r_{45}}\right)\right.
\end{aligned}
$$

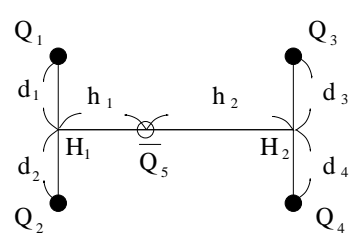

Figure 3. (a) The QQ-Q-QQ type configuration for the penta-quark system. The two QQ clusters belong to the $\mathbf{3}^{*}$ representation of the color $\mathrm{SU}(3)$. (b) A planar configuration of the pentaquark system. We take $d_{1}=d_{2}=d_{3}=d_{4} \equiv d$.

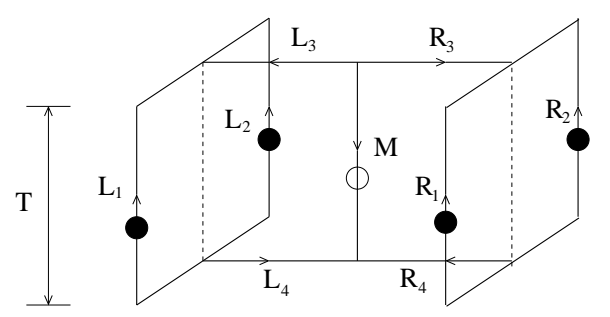

Figure 4. The penta-quark (5Q) Wilson loop $W_{5 \mathrm{Q}}$ for the $5 \mathrm{Q}$ potential $V_{5 \mathrm{Q}}$. The contours $M, L_{i}, R_{i}(i=3,4)$ are line-like and $L_{i}, R_{i}(i=$ $1,2)$ are staple-like. The $5 \mathrm{Q}$ gauge-invariant state is generated at $t=0$ and is annihilated at $t=T$.

$\left.+\frac{1}{4}\left(\frac{1}{r_{13}}+\frac{1}{r_{14}}+\frac{1}{r_{23}}+\frac{1}{r_{24}}\right)\right\}+\sigma_{5 \mathrm{Q}} L_{\min }+C_{5 \mathrm{Q}}$

with $r_{i j} \equiv\left|\mathbf{r}_{i}-\mathbf{r}_{j}\right|$ and $L_{\text {min }}$ being the minimal length of the flux-tube linking five quarks. Note that there appear three kinds of Coulomb coefficients $\left(A_{5 \mathrm{Q}}, \frac{1}{2} A_{5 \mathrm{Q}}, \frac{1}{4} A_{5 \mathrm{Q}}\right)$ in the penta-quark system, while only one Coulomb coefficient, $A_{\mathrm{Q} \overline{\mathrm{Q}}}$ or $A_{3 \mathrm{Q}}$, appears in the $\mathrm{Q} \overline{\mathrm{Q}}$ or the $3 \mathrm{Q}$ system. (In Eq.(15), $A_{5 \mathrm{Q}}$ corresponds to $A_{3 \mathrm{Q}}$ or $\frac{1}{2} A_{\mathrm{Q} \overline{\mathrm{Q}}}$ in terms of the OGE result.)

Figure 5 shows the lattice QCD results for the $5 \mathrm{Q}$ potential $V_{5 \mathrm{Q}}$. The symbols denote the lattice data, and the curves denote the theoretical form of the OGE plus multi-Y Ansatz with $\left(A_{5 \mathrm{Q}}, \sigma_{5 \mathrm{Q}}\right)$ fixed to be $\left(A_{3 \mathrm{Q}}, \sigma_{3 \mathrm{Q}}\right)$ in the $3 \mathrm{Q}$ potential $V_{3 \mathrm{Q}}$ in Ref. [7. (Note that there is no adjustable parameter for the theoretical curves besides an irrelevant constant $C_{5 \mathrm{Q}}$, since $A_{5 \mathrm{Q}}$ and $\sigma_{5 \mathrm{Q}}$ are fixed.) One finds a good agreement between the lattice data and the OGE plus multi-Y Ansatz.

We note that the multi-quark system including four or more quarks can take a three-dimensional 


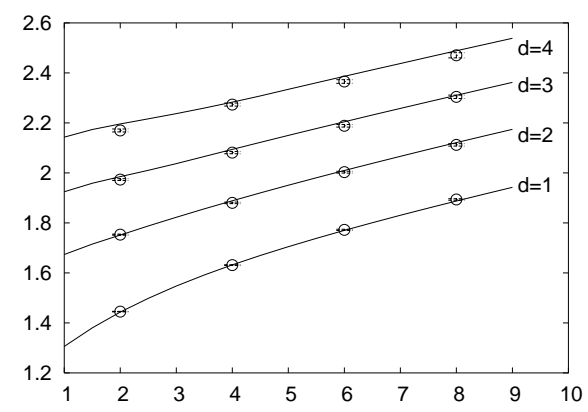

Figure 5. Lattice QCD ${ }^{\mathrm{h}}$ results of the pentaquark potential $V_{5 \mathrm{Q}}$ for the planar $5 \mathrm{Q}$ configuration with $h_{1}=h_{2} \equiv h$ in Fig.3 in the lattice unit. Each 5Q system is labeled by $d$ and $h$. The symbols denote the lattice data, and the curves the theoretical form of the OGE plus multi-Y Ansatz.

shape, while the $\mathrm{Q} \overline{\mathrm{Q}}$ and the $3 \mathrm{Q}$ systems can take only planar configuration. Then, we investigate also the twisted 5Q configuration, and find that the planar and the twisted 5Q configurations are almost degenerate. Then, no special configuration is favored in the $5 \mathrm{Q}$ system in terms of the energy, and general 5Q systems tend to take a three-dimensional configuration.

From the comparison with the QQ $\bar{Q}$ and the $3 \mathrm{Q}$ potentials 678, the universality of the string tension and the OGE result are found among $\mathrm{Q} \bar{Q}$, $3 \mathrm{Q}$ and $5 \mathrm{Q}$ systems as

$\sigma_{\mathrm{Q} \overline{\mathrm{Q}}} \simeq \sigma_{3 \mathrm{Q}} \simeq \sigma_{5 \mathrm{Q}}, \quad \frac{1}{2} A_{\mathrm{Q} \overline{\mathrm{Q}}} \simeq A_{3 \mathrm{Q}} \simeq A_{5 \mathrm{Q}}$.

This result supports the flux-tube picture on the confinement mechanism even for the multi-quark system.

\section{Gluonic Excitations in the 3Q System}

In 1969, Y. Nambu first pointed out the string picture for hadrons [18. Since then, the string picture has been one of the most important pictures for hadrons and has provided many interesting ideas in the wide region of the particle physics.

For instance, the hadronic string creates infinite number of hadron resonances as the vibrational modes, and these excitations lead to the Hagedorn "ultimate" temperature [19], which gives an interesting theoretical picture for the
QCD phase transition.

For the real hadrons, of course, the hadronic string is to have a spatial extension like the fluxtube, as the result of one-dimensional squeezing of the color-electric flux in accordance with color confinement [20]. Therefore, the vibrational modes of the hadronic flux-tube should be much more complicated, and the analysis of the excitation modes is important to clarify the underlying picture for real hadrons.

In the language of QCD, such non-quark-origin excitation is called as the "gluonic excitation", and is physically interpreted as the excitation of the gluon-field configuration in the presence of the quark-antiquark pair or the three quarks.

In the hadron physics, the gluonic excitation is one of the interesting phenomena beyond the quark model, and relates to the hybrid hadrons such as $q \bar{q} G$ and $q q q G$. In particular, the hybrid meson includes the exotic hadrons with $J^{P C}=0^{--}, 0^{+-}, 1^{-+}, 2^{+-}, \cdots$, which cannot be constructed within the simple quark model.

In this section, we study the excited-state $3 \mathrm{Q}$ potential and the gluonic excitation using lattice QCD [8], to get deeper insight on these excitations beyond the hypothetical models such as the string and the flux-tube models. Here, the excited-state $3 \mathrm{Q}$ potential is the energy of the excited state of the gluon-field configuration in the presence of the static three quarks, and the gluonic-excitation energy is expressed as the energy difference between the ground-state $3 \mathrm{Q}$ potential and the excited-state 3Q potential.

\subsection{General Formalism}

We present the formalism to extract the excited-state potential [8]. For the simple notation, the ground state is regarded as the "0th excited state". For the physical eigenstates of the QCD Hamiltonian $\hat{H}$ for the spatially-fixed 3Q system, we denote the $n$th excited state by $|n\rangle$ $(n=0,1,2, \cdots)$. Since the three quarks are spatially fixed in this case, the eigenvalue of $\hat{H}$ is expressed by a static potential as $\hat{H}|n\rangle=V_{n}|n\rangle$, where $V_{n}$ denotes the $n$th excited-state $3 \mathrm{Q}$ potential. Note that both $V_{n}$ and $|n\rangle$ are universal physical quantities relating to the QCD Hamiltonian $\hat{H}$. In fact, $V_{n}$ depends only on the $3 \mathrm{Q}$ 
location, and $|n\rangle$ satisfies the orthogonal condition as $\langle m \mid n\rangle=\delta_{m n}$.

Suppose that $\left|\Phi_{k}\right\rangle(k=0,1,2, \cdots)$ are arbitrary given independent spatially-fixed $3 \mathrm{Q}$ states. In general, each $3 Q$ state $\left|\Phi_{k}\right\rangle$ can be expressed by a linear combination of $3 \mathrm{Q}$ physical eigenstates $|n\rangle$,

$$
\left|\Phi_{k}\right\rangle=c_{0}^{k}|0\rangle+c_{1}^{k}|1\rangle+c_{2}^{k}|2\rangle+\cdots .
$$

Here, the coefficients $c_{n}^{k}$ depend on the selection of $\left|\Phi_{k}\right\rangle$, and hence they are not universal quantities.

The Euclidean-time evolution of the $3 \mathrm{Q}$ state $\left|\Phi_{k}(t)\right\rangle$ is expressed with the operator $e^{-\hat{H} t}$, which corresponds to the transfer matrix in lattice QCD. The overlap $\left\langle\Phi_{j}(T) \mid \Phi_{k}(0)\right\rangle$ is given by the $3 \mathrm{Q}$ Wilson loop with the initial state $\left|\Phi_{k}\right\rangle$ at $t=0$ and the final state $\left|\Phi_{j}\right\rangle$ at $t=T$, and is expressed in the Euclidean Heisenberg picture as

$$
\begin{aligned}
& W_{T}^{j k} \equiv\left\langle\Phi_{j}\left|W_{3 \mathrm{Q}}(T)\right| \Phi_{k}\right\rangle=\left\langle\Phi_{j}\left|e^{-\hat{H} T}\right| \Phi_{k}\right\rangle \\
& =\sum_{m=0}^{\infty} \sum_{n=0}^{\infty} \bar{c}_{m}^{j} c_{n}^{k}\left\langle m\left|e^{-\hat{H} T}\right| n\right\rangle=\sum_{n=0}^{\infty} \bar{c}_{n}^{j} c_{n}^{k} e^{-V_{n} T} .
\end{aligned}
$$

Using the matrix $C$ satisfying $C^{n k}=c_{n}^{k}$ and the diagonal matrix $\Lambda_{T}$ as $\Lambda_{T}^{m n}=e^{-V_{n} T} \delta^{m n}$, we rewrite the above relation as

$W_{T}=C^{\dagger} \Lambda_{T} C$.

Note here that $C$ is not a unitary matrix, and hence this relation does not mean the simple diagonalization by the unitary transformation.

Since we are interested in the $3 \mathrm{Q}$ potential $V_{n}$ in $\Lambda_{T}$ rather than the non-universal matrix $C$, we single out $V_{n}$ from the $3 \mathrm{Q}$ Wilson loop $W_{T}$ as

$W_{T}^{-1} W_{T+1}=C^{-1} \operatorname{diag}\left(e^{-V_{0}}, e^{-V_{1}}, \cdots\right) C$,

which is a similarity transformation. Then, $e^{-V_{n}}$ can be obtained as the eigenvalues of the matrix $W_{T}^{-1} W_{T+1}$, i.e., solutions of the secular equation,

$\operatorname{det}\left\{W_{T}^{-1} W_{T+1}-t \mathbf{1}\right\}=\prod_{n}\left(e^{-V_{n}}-t\right)=0$.

Thus, the $3 \mathrm{Q}$ potential $V_{n}$ can be obtained from the matrix $W_{T}^{-1} W_{T+1}$.

In the practical calculation, we prepare $N$ independent sample states $\left|\Phi_{k}\right\rangle(k=0,1, \cdots, N-1)$. By choosing appropriate states $\left|\Phi_{k}\right\rangle$ so as not to include highly excited-state components, the physical states $|n\rangle$ can be truncated as $0 \leq n \leq$ $N-1$. Then, $W_{T}, C$ and $\Lambda_{T}$ are reduced into $N \times N$ matrices, and the secular equation (11) becomes the $N$ th order equation.

\subsection{Lattice QCD for Gluonic Excitations}

For about 100 different patterns of spatiallyfixed 3Q systems, we calculate the excited-state potential using $\mathrm{SU}(3)$ lattice $\mathrm{QCD}$ with $16^{3} \times 32$ at $\beta=5.8$ and 6.0 at the quenched level [8]. In Fig.6, we show the 1st excited-state $3 \mathrm{Q}$ potential $V_{3 \mathrm{Q}}^{\text {e.s. }}$ and the ground-state potential $V_{3 \mathrm{Q}}^{\text {g.s. }}$.
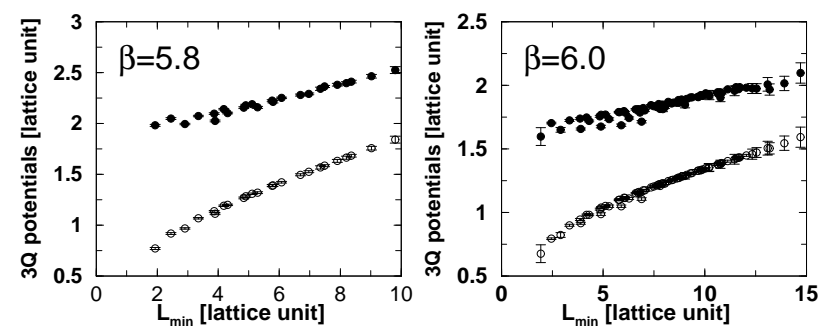

Figure 6 . The 1 st excited-state $3 \mathrm{Q}$ potential $V_{3 \mathrm{Q}}^{\text {e.s. }}$ and the ground-state $3 \mathrm{Q}$ potential $V_{3 \mathrm{Q}}^{\mathrm{g} . \mathrm{s}}$. The lattice results at $\beta=5.8$ and $\beta=6.0$ well coincide besides an irrelevant overall constant. The gluonic excitation energy $\Delta E_{3 \mathrm{Q}} \equiv V_{3 \mathrm{Q}}^{\mathrm{e} . \mathrm{s}}-V_{3 \mathrm{Q}}^{\mathrm{g} . \mathrm{s}}$ is found to be about $1 \mathrm{GeV}$ in the hadronic scale.

The energy gap between $V_{3 \mathrm{Q}}^{\text {g.s. }}$ and $V_{3 \mathrm{Q}}^{\text {e.s. }}$ physically means the excitation energy of the gluonfield configuration in the presence of the spatiallyfixed three quarks, and the gluonic excitation energy $\Delta E_{3 \mathrm{Q}} \equiv V_{3 \mathrm{Q}}^{\text {e.s. }}-V_{3 \mathrm{Q}}^{\text {g.s. }}$ is found to be about $1 \mathrm{GeV} 817$ in the hadronic scale as $L_{\min } \sim 1 \mathrm{fm}$.

Note that the gluonic excitation energy of about $1 \mathrm{GeV}$ is rather large in comparison with the excitation energies of the quark origin. The present result predicts that the lowest hybrid baryon, which is described as $q q q G$ in the valence picture, has a large mass of about $2 \mathrm{GeV}$ 8 17. (The present result seems to suggest the constituent gluon mass of about $1 \mathrm{GeV}$.)

\section{Behind the Success of the Quark Model}

Finally, we consider the connection between QCD and the quark model in terms of the gluonic excitation 817. While QCD is described with 
quarks and gluons, the simple quark model successfully describes low-lying hadrons even without explicit gluonic modes. In fact, the gluonic excitation seems invisible in the low-lying hadron spectra, which is rather mysterious.

On this point, we find the gluonic-excitation energy to be about $1 \mathrm{GeV}$ or more, which is rather large compared with the excitation energies of the quark origin, and therefore the effect of gluonic excitations is negligible and quark degrees of freedom plays the dominant role in low-lying hadrons with the excitation energy below $1 \mathrm{GeV}$.

Thus, the large gluonic-excitation energy of about $1 \mathrm{GeV}$ gives the physical reason for the invisible gluonic excitation in low-lying hadrons, which would play the key role for the success of the quark model without gluonic modes 817].

In Fig.7, we present a possible scenario from QCD to the massive quark model in terms of color confinement and dynamical chiralsymmetry breaking (DCSB) [17.

\section{REFERENCES}

1. Y. Nambu, in Preludes in Theoretical Physics, (North-Holland, Amsteldam, 1966).

2. M.Y. Han and Y. Nambu, Phys. Rev. 139 (1965) B1006.

3. Articles in Color Confinement and Hadrons in Quantum Chromodynamics, edited by H. Suganuma et al. (World Scientific, 2004).

4. Y. Nambu, G. Juna-Lasinio, Phys. Rev. 122 (1961) 345; ibid. 124 (1961) 246.

5. H.J. Rothe, Lattice Gauge Theories, 2nd edition (World Scientific, 1997) p.1.

6. T.T. Takahashi, H. Matsufuru, Y. Nemoto, H. Suganuma, Phys. Rev. Lett. 86 (2001) 18.

7. T.T. Takahashi, H. Suganuma, Y. Nemoto, H. Matsufuru, Phys.Rev. D65 (2002) 114509.

8. T.T. Takahashi and H. Suganuma, Phys. Rev. Lett. 90 (2003) 182001.

9. T.T. Takahashi, H. Matsufuru, Y. Nemoto and H. Suganuma, Dynamics of Gauge Fields, Tokyo, Dec. 1999, edited by A. Chodos et al., (Universal Academy Press, 2000) 179; H. Suganuma, Y. Nemoto, H. Matsufuru and T.T. Takahashi, Nucl.Phys. A680 (2000) 159.

10. J. Kogut, L. Susskind, Phys. Rev. D11 (1975)
395; J. Carlson, J. Kogut, V. Pandharipande, Phys. Rev. D27 (1983) 233; D28 (1983) 2807.

11. C. Alexandrou, P. de Forcrand, A. Tsapalis, Phys. Rev. D65 (2002) 054503.

12. O. Jahn and P. de Forcrand, Nucl. Phys. B (Proc. Suppl.) 129 (2004) 700.

13. D.S. Kuzmenko and Yu.A. Simonov, Phys. Atom. Nucl. 66 (2003) 950.

14. J.M. Cornwall, Phys. Rev. D54 (1996) 6527.

15. J.M. Cornwall, Phys.Rev.D69 (2004) 065013.

16. H. Ichie, V. Bornyakov, T. Streuer and G. Schierholz, Nucl. Phys. A721 (2003) 899; Nucl. Phys. B (Proc.Suppl.) 119 (2003) 751.

17. H. Suganuma, T.T. Takahashi and H. Ichie, Color Confinement and Hadrons in Quantum Chromodynamics, (World Scientific, 2004) p.249; Nucl. Phys. A (2004).

18. Y. Nambu, in Symmetries and Quark Models (Wayne State University, 1969); Lecture Notes at the Copenhagen Symposium (1970). 19. R. Hagedorn, Nuovo Cim.Suppl. 3 (1965) 147. 20. Y. Nambu, Phys. Rev. D10 (1974) 4262. 
Quantum Chromodynamics

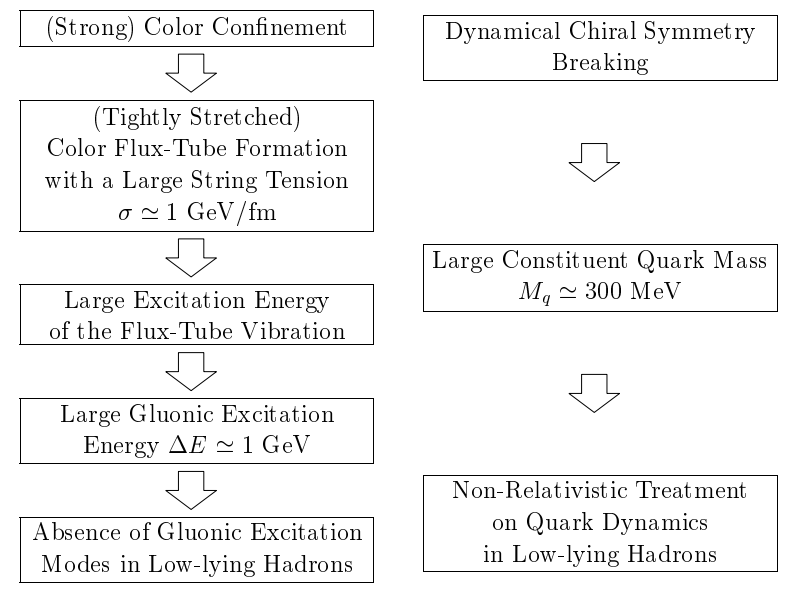

Massive Quark Model for Low-lying Hadrons

Figure 7. A possible scenario from QCD to the quark model in terms of color confinement and DCSB. DCSB provides a large constituent quark mass of about $300 \mathrm{MeV}$, which enables the nonrelativistic treatment for quark dynamics. Color confinement leads to the color flux-tube formation among quarks. In the flux-tube picture, the gluonic excitation is described as the flux-tube vibration, and then its energy is expected to be large in the hadronic scale. The large gluonicexcitation energy of about $1 \mathrm{GeV}$ leads to the absence of the gluonic mode in low-lying hadrons, which would play the key role to the success of the quark model without gluonic excitation modes. 